\title{
Temperature Measurement in a THM Solution Zone for the Growth of $\mathrm{Hg}_{1-x} \mathrm{Cd}_{x} \mathrm{Te}$
}

\begin{abstract}
A combined process for temperature measurements in THM growth systems is described including the preparation of the source material as well as the liquid solution zone in one and the same ampoule. Using starting compositions with an excess of that component to act as the solvent the solution zone is formed during the travelling heater procedure. With thermocouples attached to the slightly modified ampoule axial temperature distributions were recorded under the usual THM growth conditions. The measurements were done in $\mathrm{Hg}_{0.8} \mathrm{Cd}_{0.2}$ Te growing from $\mathrm{Te}$-rich solution zones. The procedure described can be applied to all materials for which THM crystal growth can be achieved.
\end{abstract}

Temperaturmessungen in THM-Kristallzüchtungsanordnungen werden beschrieben, die einen kombinierten Prozeß der Herstellung des Quellenmaterials und der Lösungszone einschließen, der in einer einzigen Ampulle erfolgt. Ausgehend von einem Überschuß der als Lösungsmittel wirkenden Komponente, bildet sich die Lösungszone während der üblichen Heizertranslation. Thermoelemente, die sich an der nur wenig modifizierten Ampulle befinden, registrieren die axiale Temperaturverteilung unter Bedingungen, wie sie für die THM-Züchtung üblich sind. Die Messungen wurden bei der Kristallzüchtung von $\mathrm{Hg}_{0.8} \mathrm{Cd}_{0.2} \mathrm{Te}$ aus Te-reichen Lösungszonen durchgeführt. Die beschriebene Variante ist auf alle anderen Materialien, die nach dem THM-Verfahren gezüchtet werden können, ebenfalls anwendbar.

\section{Introduction}

The travelling heater method (THM) is a special crystal growth technique which has been successfully applied to the growth of single crystals of several II-VI and III-V compounds and alloys. This technique has been particularly well accepted as a method of growing high-purity and homogeneous mixed crystals of $\mathrm{Hg}_{1-x} \mathrm{Cd}_{x} \mathrm{Te}$ (TRIBOULET et al. 1985) and $\mathrm{Hg}_{1-x} \mathrm{Zn}_{x}$ Te (TRIBOULET et al. 1986). This is caused by the principle of THM which combines the steady-state conditions of the zone melting technique with the low-temperature growth of crystallization from solution.

In the travelling heater method the crystal grows by passing a solution zone (e.g. Te-rich) through a polycrystalline rod (e.g. $\mathrm{Hg}_{1-x} \mathrm{Cd}_{x} \mathrm{Te}$ ) of the composition to grow. The motion of the zone is produced by the motion of the heater. The crystal growth rate is strongly limited by the material transport through the liquid zone. For the growth of the above mentioned crystals and usual geometries convection appears to be the dominant mechanism. Convection as well as diffusion within the boundary layers attached to the two liquid/solid interfaces are influenced by the axial temperature distribution. It must be exactly known before THM crystal growth conditions can be reliably set by other than trial and error techniques.

In literature, there are known different ways to get information about the temperature distribution in THM solution zones. First, there are some attempts to calculate temperature 
fields within the liquid zone as well as the adjacent crystalline rods (BELL; WALD, BeLL; YIP et al.; CherePanova). Besides the relatively high effort which is necessary to gain exact results, this way suffers from a noticeable lack in the literature of data of the systems under consideration in the interesting temperature region.

The other way is the experimental determination of axial temperature distributions in arrangements being as similar as possible to the actual THM crystal growth parameters. It is done e.g. with rods of special materials having the same thermal conductivity as the crystal to grow and carrying thermocouples for measuring the temperature at certain positions or by moving the heater over the thermocouple at fixed position (TRIBOULET). This method neglects the special behaviour of the actual solution zone with respect to thermodiffusive and thermo-convective data of the liquid, being different from those of the solid material.

A better but much more complicated way of measuring temperature distributions is to get information from the THM-identical arrangement consisting of the material to grow including the liquid zone but with an additional thermocouple in the crystal axis (SCHWENKENBECHER, RUDOLPH). This method presents several drawbacks like successive handling of the ingot and the zone material which have to be cut and drilled for the mechanical adjustment of the thermocouple. This irksome procedure is connected with the resulting risks of pollution and of producing cavities. But dealing with Hg-containing alloys every kind of empty space within the arrangement should be prevented. This is true of THM crystal growth as well as of the temperature-measuring experiments.

That is, why we prepared a THM-identical growth arrangement with a $\mathrm{Hg}_{1-x} \mathrm{Cd}_{x} \mathrm{Te}$ rod, a Te-rich solution zone and a thermocouple in the axis of the ingot in one and the same ampoule without any handling of the material.

In the specially designed ampoule a non-stoichiometric mixture of $\left(\mathrm{Hg}_{1-x_{0}} \mathrm{Cd}_{x_{0}}\right)_{1-y_{0}}, \mathrm{Te}_{y_{0}}$, $\left(y_{0}>0.5\right)$ is to be homogenized and quenched. By moving just this ampoule through the THM ring heater the Te excess will be continuously collected thus forming the usual solution zone. This process is to be calculated in order to produce the interesting solution zone length just when passing the fixed position of the thermocouple. The temperature versus time measurement can be transformed into the axial temperature distribution by the known translation rate. This method is not limited to the $\mathrm{Hg}_{1-x} \mathrm{Cd}_{x} \mathrm{Te}$ system but is of general interest and could be applied to all materials for which THM crystal growth can be achieved.

\section{Theoretical}

The starting non-stoichiometric composition $\left(\mathrm{Hg}_{1-x_{0}} \mathrm{Cd}_{x_{0}}\right)_{1-x_{0}} \mathrm{Te}_{y_{0}}$ with $y_{0}>0.5$ forms after homogenization and quenching an ingot consisting of two phases which portions correspond to the initial mole fraction $y_{0}:\left(1-y_{0}\right) \mathrm{Hg}_{1-x_{0}} \mathrm{Cd}_{x_{0}} \mathrm{Te}$ and $\left(2 y_{0}-1\right) \mathrm{Te}$. Quenching should be carried out in such a way, that the two phases being homogeneously distributed in a macroscopic scale. Passing the ingot through the temperature field of the ring heater the liquid droplets of the Te phase will be collected forming a liquid zone of the composition $\left(\mathrm{Hg}_{1-z} \mathrm{Cd}_{z}\right)_{1-y} \mathrm{Te}_{y}$ (TILLER) (see Fig. 1).

The mole fraction $y$ of the solution zone is determined by the temperature of the phase boundaries which is to be influenced by the temperature of the furnace used. 
Fig. 1. Principle scheme of the various stages of the THM procedure for temperature measurements. a) After having quenched the melt the ingot consists of the $(\mathrm{Hg}, \mathrm{Cd}) \mathrm{Te}$ matrix and homogeneously distributed Te inclusions; b) and c) During the THM process the Te inclusions from a continuously broadening solution zone

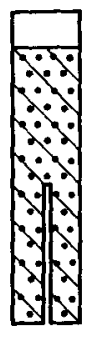

a)

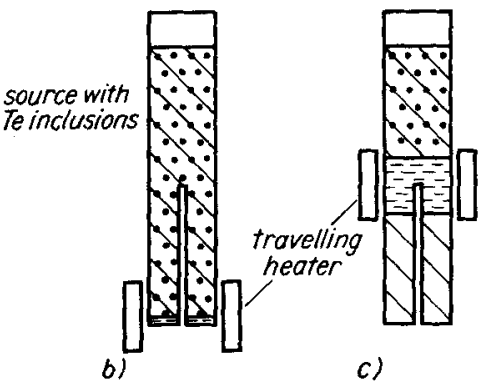

The length $l$ of the liquid solution zone at a certain axial position $d$ of the ingot consists of a fictive length $l_{0}$ corresponding to the amount of the pure solvent Te and the difference to $l$ which is formed by the alloy $\mathrm{Hg}_{1-z} \mathrm{Cd}_{z} \mathrm{Te}$ being solved in it. The continuously with $d$

Fig. 2. Sketch of the ingot with the solution zone to explain the geometrical terms used in calculation

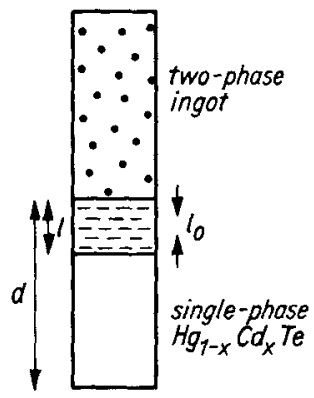

increasing length $l_{0}$ of pure Te is easily to be calculated when assuming a constant cross section of the ingot. The ratio $l_{0} / d$ is constant and determined only by the volume fraction of excess Te compared to the volume of the total ingot given by the mole fraction $y_{0}$ of the initial charge:

$$
\frac{l_{0}}{d}=\frac{1}{1+\frac{1-y_{0}}{2 y_{0}-1} f}, \text { with } f=\frac{M_{\mathrm{MCT}} \varrho_{\mathrm{Te}}}{\varrho_{\mathrm{MCT}} M_{\mathrm{Te}}} .
$$

With $M_{\mathrm{i}}$ being the molar masses of $(\mathrm{Hg}, \mathrm{Cd}) \mathrm{Te}(\mathrm{MCT})$ and $\mathrm{Te}$, and $\varrho_{\mathrm{i}}$ being the corresponding densities, $f$ is the ratio of molar volumes of $(\mathrm{Hg}, \mathrm{Cd}) \mathrm{Te}$ and $\left.\mathrm{Te}^{1}\right)$. The given amount of pure Te corresponding to the length $l_{0}$ solves a certain quantity of the crystalline material adjacent to it, thus forming the solution zone of the length $l$. By reason of the balance of material the ratio $l_{0} / l$ only depends on the mole fraction $y$ of the solution zone determined by the temparature of the interface:

$$
\frac{l_{0}}{l}=\frac{1}{1+\frac{1-y}{2 y-1} f}, \text { with } y=y(T) .
$$

1) Within the frame of this calculation the ratio of the molar volumes $f$ is regarded to be independent on $x$. Generally, the assumption of nearly constant molar volumes is a good approximation in the most solid solution systems. With $(\mathrm{Hg}, \mathrm{Cd}) \mathrm{Te}$ it is particularly well met because of the low difference between the lattice parameters of $\mathrm{HgTe}$ and CdTe. 
From equs 1 and 2 it follows how the length of the liquid solution zone increases with the axial distance $d$ of the ingot passed by:

$$
l=\frac{1+\frac{1-y}{2 y-1} f}{1+\frac{1-y_{0}}{2 y_{0}-1} f} d
$$

With solid/liquid phase boundaries of constant temperature (i.e. $y(T)=$ const.) the length of the liquid solution zone increases linearly with the axial position. The mole fraction $y_{0}$ of the initial charge can now be calculated in such a way that for the aim of the temperature measurement the solution zone reachs just at the position of the fixed thermocouples that length, which is typical of THM crystal growth conditions. Figure 3 represents the conditions necessary to produce a solution zone from a Te-rich starting material $\left(\mathrm{Hg}_{0.78} \mathrm{Cd}_{0.22}\right)_{1-y_{0}} \mathrm{Te}_{y_{0}}$. Solubility data are taken from the numerical description of the phase diagram given by BRICE.

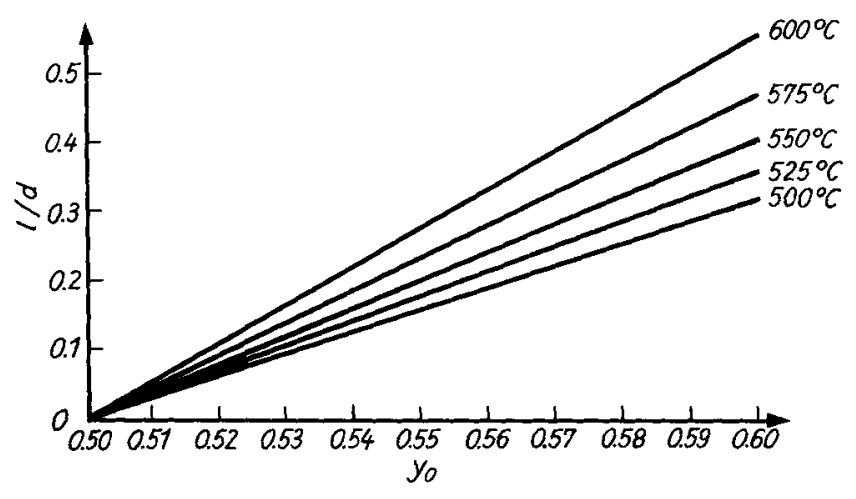

Fig. 3. Broadening rate $l / d$ versus original mole fraction $y_{0}$ representing the Te excess. The parameter $T$ corresponds to the interface temperature of the solution zone. Use was made of the solubility data $y_{0}(T)$ according to BRICE

$\left(\mathrm{Hg}_{0.78} \mathrm{Cd}_{0.22}\right)_{1-y_{0}} \mathrm{Te}_{y_{0}}$

\section{Experimental}

A specially designed quartz ampoule (see Fig. 4) with an inner diameter of $13 \mathrm{~mm}$ and $2 \mathrm{~mm}$ thick walls was used for temperature measurements. The ampoule contained a quartz capillary of an outer diameter of $3 \mathrm{~mm}$ to cover a $0.5 \mathrm{~mm}$ diameter $\mathrm{Ni}-\mathrm{CrNi}$ thermocouple. Considering problems of heat conduction, the cross section of the ingot was correct with the exception of $5 \%$ which was occupied by the capillary for the thermocouple. The starting material was previously synthesized $\mathrm{HgTe}, \mathrm{CdTe}$ and multi-zone-refined $\mathrm{Te}$ as to yield a $103 \mathrm{~mm}$ long ingot of an average composition of $\left(\mathrm{Hg}_{1-x_{0}} \mathrm{Cd}_{x_{0}}\right)_{1-y_{0}} \mathrm{Te}_{y_{0}}$ with $x_{0}=0.195$ and $y_{0}=0.540$. After homogenization at $800^{\circ} \mathrm{C}$ for 60 hours in a horizontal position the ampoule was vertically quenched by lowering it with a rate as high as $2.5 \mathrm{~m} \mathrm{~h}^{-1}$ in a conventional furnace.

The quenched ampoule was change to the THM furnace without opening or any material handling but attaching the two thermocouples at those positions pictured in Fig. 4. The heater moved upwards with a constant translation rate of $2.2 \mathrm{~mm} \mathrm{~d}^{-1}$ which is somewhat higher than in usual THM crystal growth runs but low enough to collect all the Te inclusions corresponding to the excess of the starting material. While moving the heater with respect to the fixed ampoule the temperatures at the two thermocouples were recorded. With ingots being long enough temperature versus time relations at fixed positions but with a travelling solution zone may be interpreted as axial temperature distributions by the help of a known translation rate. 
Fig. 4. Detailed picture of the special THM ampoule to measure temperatures within the material during growth

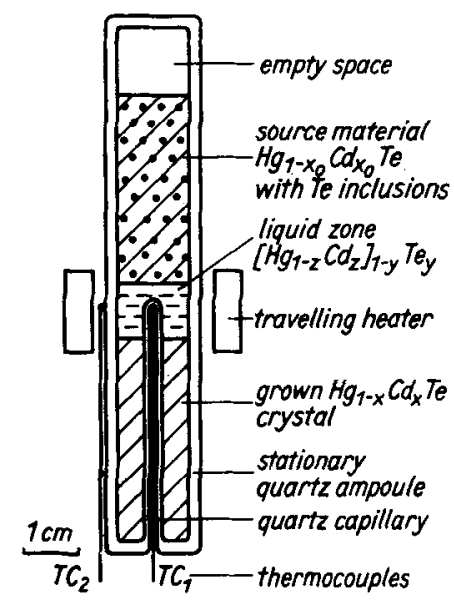

\section{Results and discussion}

The measured axial temperature distributions recorded by the inner as well as the outer thermocouple are pictured in Figure 5. The left-hand side of the picture represents the upper part of the THM arrangement (source material) while the right-hand side stands for the first grown crystal. Identification of the solid/liquid phase boundary positions has been done by the help of the more detailed plots of the measured temperature distribution (thermocouple 1) which show distinct changes of the gradients at the interfaces. The outer thermocouple does not record clear modifications when passing the phase boundaries because of the influence of the surrounding medium.

Fig. 5. Measured axial temperature distributions (along the crystal axis and next to the ampoule wall) in the THM arrangement of a $(\mathrm{Hg}, \mathrm{Cd}) \mathrm{Te}$ crystal growing from its Te-rich solution zone

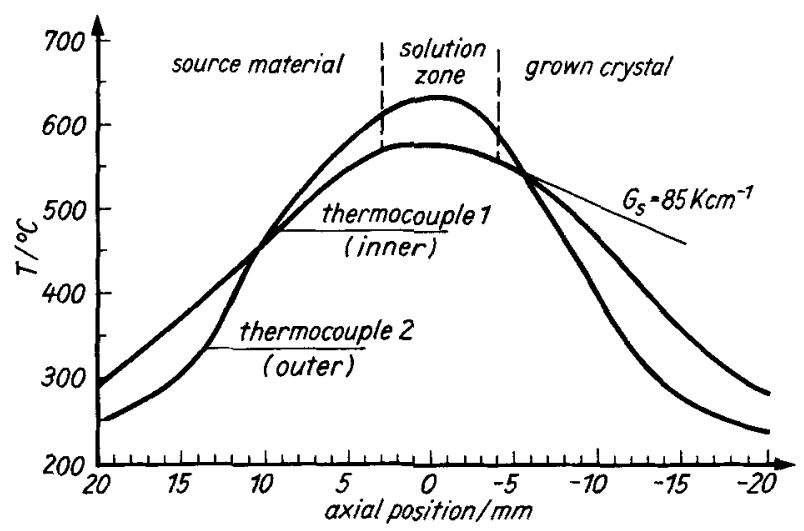

What is interesting are the points of intersection of the two temperature distributions. They mark those axial positions where flat isotherms are to be assumed. While the plot of the temperature distribution recorded by the inner thermocouple shows a nearly symmetric behaviour with respect to the temperature maximum, the temperatures measured by the outer thermocouple are higher in the upper part influenced by the convection of the surrounding medium. That is why the intersection in the upper region of the THM arrangement has been drifted upwards. For all interface positions between the two points 
of intersection convex shapes of the phase boundaries with respect to the solid phases are to be expected becoming more convex with smaller zone length. Along the axis of the ampoule the liquid zone has been measured to be $7 \mathrm{~mm}$ long. Assuming the phase boundaries to be isotherms the distance between the solid/liquid interfaces reachs about $10 \mathrm{~mm}$ near the wall of the ampoule.

It is well known from the principle of mass transport in THM that the temperature difference between the dissolving and growing interfaces is produced by the motion of the heater and the limited diffusion rate in the liquid zone. With a furnace translation rate of $2.2 \mathrm{~mm} \mathrm{~d}^{-1}$ the temperature difference between the two phase boundaries has been recorded to be $12 \mathrm{~K}$ with an interface temperature of the growing crystal of $558^{\circ} \mathrm{C}$.

The axial temperature gradient in the growing crystal next to the solid/liquid interface has been measured to be as high as $85 \mathrm{~K} \mathrm{~cm}^{-1}$ becoming even higher with increasing distance from the phase boundary. High axial temperature gradients are necessary for material transport in THM but rather detrimental to the structural perfection of the growing crystal when accompanied by nonuniformity.

During the measurements with the inner thermocouple at positions within the liquid zone some sort of periodic temperature fluctuations have been registered. The preparation technique described in this paper offers unique possibilities to detect it under THM-identical conditions. Further investigations should be carried out to allow detailed interpretation.

\section{Conclusion}

Temperature distributions were measured within a THM ampoule during the growth of $(\mathrm{Hg}, \mathrm{Cd}) \mathrm{Te}$ from a Te-rich solution zone. Contrary to what is known from literature, the whole arrangement consisting of two solid ingots with a liquid solution zone between them was created during one combined procedure of quenching and zone processes from a non-stoichiometric melt. The results of the measurement reveal axial temperature profiles which are necessary to know for setting optimum crystal growth conditions. The method may be applied to all THM crystal growth problems.

\section{References}

BeLL, R. O.: J. Electrochem. Soc. 121 (1974) 1366

BRICE, J. C.: Progr. Cryst. Growth Charact. 13 (1986) 39

Cherepanova, T. A.: Cryst. Res. Technol. 17 (1982) 735

SCHWENKENBECHER, K., RuDOLPH, P.: Cryst. Res. Technol. 20 (1985) 1609

Tiller, W. A.: J. Appl. Physics 34 (1963) 2757; 34 (1963) 2763; 36 (1965) 261

TRIBOULET, R.: private communication

Triboulet, R., Nguyen Duy, T., Durand, A.: J. Vac. Sci. Technol. A3 (1985) 95

Triboulet, R., Lasbley, A., Toulouse, B., Granger, R.: J. Cryst. Growth 79 (1986) 695

Wald, F. V., Bell, R. O.: J. Cryst. Growth 30 (1975) 29

YiP, V. F. S., ChanG, C. E., Wilcox, W. R.: J. Cryst. Growth 29 (1975) 69

(Received April 5, 1990 accepted April 16, 1990)

Author's address:

Dipl.-Krist. F. M. Kiessuing, Dr. rer. nat. P. Gille

Humboldt-Universität zu Berlin

Sektion Physik, Bereich Kristallographie

Invalidenstr. 110

O-1040 Berlin 